\title{
Surface drift prediction in the Adriatic Sea using hyper-ensemble statistics on atmospheric, ocean and wave models: Uncertainties and probability distribution areas
}

\author{
M. Rixen ${ }^{\mathrm{a}, *}$, E. Ferreira-Coelho ${ }^{\mathrm{a}, 1}$, Richard Signell ${ }^{\mathrm{b}, 2}$ \\ ${ }^{a}$ NATO Undersea Research Centre (NURC), Viale San Bartolomeo 400, 19026 La Spezia (SP), Italy \\ ${ }^{\mathrm{b}}$ US Geological Survey, 384 Woods Hole Road, Woods Hole, MA 02543-1598, USA
}

Available online 20 February 2007

\begin{abstract}
Despite numerous and regular improvements in underlying models, surface drift prediction in the ocean remains a challenging task because of our yet limited understanding of all processes involved. Hence, deterministic approaches to the problem are often limited by empirical assumptions on underlying physics. Multi-model hyper-ensemble forecasts, which exploit the power of an optimal local combination of available information including ocean, atmospheric and wave models, may show superior forecasting skills when compared to individual models because they allow for local correction and/or bias removal. In this work, we explore in greater detail the potential and limitations of the hyper-ensemble method in the Adriatic Sea, using a comprehensive surface drifter database. The performance of the hyper-ensembles and the individual models are discussed by analyzing associated uncertainties and probability distribution maps. Results suggest that the stochastic method may reduce position errors significantly for 12 to $72 \mathrm{~h}$ forecasts and hence compete with pure deterministic approaches.
\end{abstract}

(C) 2007 NATO Undersea Research Centre (NURC). Published by Elsevier B.V. All rights reserved.

Keywords: Forecast; Surface drift; Multi-model super-ensembles; Linear regression; Ocean models; Atmospheric models; Wave models

\section{Introduction}

A plethora of ocean, wave and atmospheric models are currently available on a routine basis at the global, regional and local scale in many coastal areas. Funda-

\footnotetext{
* Corresponding author. Tel.: +39 0187527 457; fax: +39 0187527 354.

E-mail addresses: rixen@nurc.nato.int (M. Rixen), coelho@nrlssc.navy.mil (E. Ferreira-Coelho),rsignell@usgs.gov (R. Signell).

${ }^{1}$ Naval Research Laboratory, Code 7322, Bldg 1009, Room C128, Stennis Space Center, MS39529, USA. Tel.: +1 228688 5710; fax: +1 2286884759 .

${ }^{2}$ Tel.: +1 508457 2229; fax: +1 5084572310 .
}

mental questions arise concerning which model to select for a given task, what criterion to apply for this selection and what is the resulting confidence level. All such models have varying skills in space, in time, but also in frequency. A master model may introduce direct errors on a slave model in a one-way coupled implementation or even feedback errors on itself in two-way coupled implementations, generating a complex chain of errors known as the "uncertainty cascade".

Much effort is spent on individual model improvements, limited at a point beyond which processes have to be simulated in a non-deterministic way. An original statistical approach was recently proposed to circumvent this limitation, aimed at combining optimally different models into a super-ensemble for weather and climate 
forecast (Krishnamurti et al., 2000a,b; Kumar et al., 2003; Shin and Krishnamurti, 2003a,b) using least squares optimization, dynamic linear models and probabilistic approaches.

These techniques have been also successfully applied in the ocean for sound velocity profile estimations (Rixen and Ferreira-Coelho, 2005) and for surface drift problems (Rixen and Ferreira-Coelho, in press).

Surface drift prediction can be very challenging in certain areas because of the number, and the complex interplay, of processes involved (e.g. Carniel et al., 2002; Rixen and Ferreira-Coelho, in press), including Ekman transport, tides, Stokes drift, ocean currents, inertial oscillation, leeway effects, etc. As a rule of thumb, Ekman drift will set up a surface current of roughly $\sim 3 \%$ of the wind speed, $\sim 15^{\circ}$ to the right of the downwind direction in the Northern hemisphere. But these values may vary according to the sea state and the stratification (e.g. Gill, 1982). Other processes may have similar contributions to the flow, such as the effect of waves and ocean. Deterministic methods do not yet exist to combine these effects, and it is hence natural to try non-deterministic or statistical approaches to solve surface drift problems.

In the present study, the hyper-ensemble approach developed in Rixen and Ferreira-Coelho (in press) is applied: (1) to forecast at short time scale surface drifts from combined atmospheric ocean, wave models and local drifter observations in the Adriatic during a "Bora" event that occurred in February 2003, a wind responsible for deep water formation in the area in winter (e.g. Signell et al., 2005); and (2) to derive uncertainty/ probability areas for drifter positions. Data, models and the hyper-ensemble methodology are detailed in Section 2. Results are presented in Section 3 and conclusions are drawn in Section 4.

\section{Data and models}

\subsection{Data - drifters}

Lagrangian drifters provide a broad basin-scale coverage of mesoscale surface circulation and surface temperatures to study the movement of water masses (e.g. Kovacevic et al., 1999; Poulain et al., 2003). Typical drifters that track the top one-meter of the ocean surface show excellent coupling to the surface layer and exhibit little wave rectification. During field experiments ADRIA02 and ADRIA03 with the R/V ALLIANCE between fall 2002 and spring 2003, some 144 drifters were launched in the Adriatic, building the comprehensive database used in this study (Fig. 1).

\subsection{The atmospheric, ocean and wave models}

\subsubsection{ROMS circulation model}

To simulate near-surface ocean circulation, the Regional Ocean Modeling System (ROMS) version 2.1 was used. This model was selected because it has several features of potential benefit for the study of near-surface currents. The $s$-coordinate allows more flexibility than the sigma coordinate in specifying vertical grid spacing, allowing thin layers near the surface to have a more uniform thickness. In addition, version 2.1 contains the Generic Length Scale (GLS) mixing scheme of Umlauf and Burchard (2003), which can be configured with parameters that allow the model to represent the correct dissipation profile under strong wind driving with breaking surface waves. The model was configured in curvilinear coordinates with variable grid resolution ranging from 3 to $4 \mathrm{~km}$ in the northern Adriatic to 7$9 \mathrm{~km}$ in the southern Adriatic. The model was initialized in mid-September 2002 using in situ observations and driven with tides and no-gradient temperature and salinity open boundary conditions at the narrow entrance to the Adriatic Sea. Wind, air temperature, air pressure, cloud fraction, short-wave radiation and relative humidity were used from LAMI (see below) with sea surface temperature from ROMS to compute bulk momentum and heat fluxes using the COARE 2.6 algorithms. The model was run from September 17, 2002 to June 13, 2003, and output saved every $3 \mathrm{~h}$. For further details on the model implementation, see Signell et al. (2005) and references therein.

\subsubsection{LAMI model}

LAMI (Limited Area Model Italy) is the Italian operational implementation of LOKAL MODELL, the limited area model originally developed by the German Meteorological Service (Deutscher WetterDienst, DWD) for meso/micro scale weather prediction and simulation developed by several European meteorological services belonging to COSMO (COnsortium for Small scale MOdelling). LAMI is managed by SMR-ARPA-EMR, UGM (Ufficio Generale per la Meteorologia, Italian Airforce) and Regione Piemonte. It has been operational since the beginning of 2001 at the CINECA supercomputing Centre in Bologna. It has a $7 \mathrm{~km}$ grid spacing and 35 vertical terrain-following levels. It is a fully compressible, non-hydrostatic 3D model in which initial and boundary conditions are obtained from the DWD global circulation model GME (Majewsky, 1998; Majewsky et al., 2002). LAMI gives output every $3 \mathrm{~h}$ and produces a 48 -hour forecast daily. We therefore used forecast winds at $03,06,09, \ldots 24 \mathrm{~h}(00+03,00+06$, 


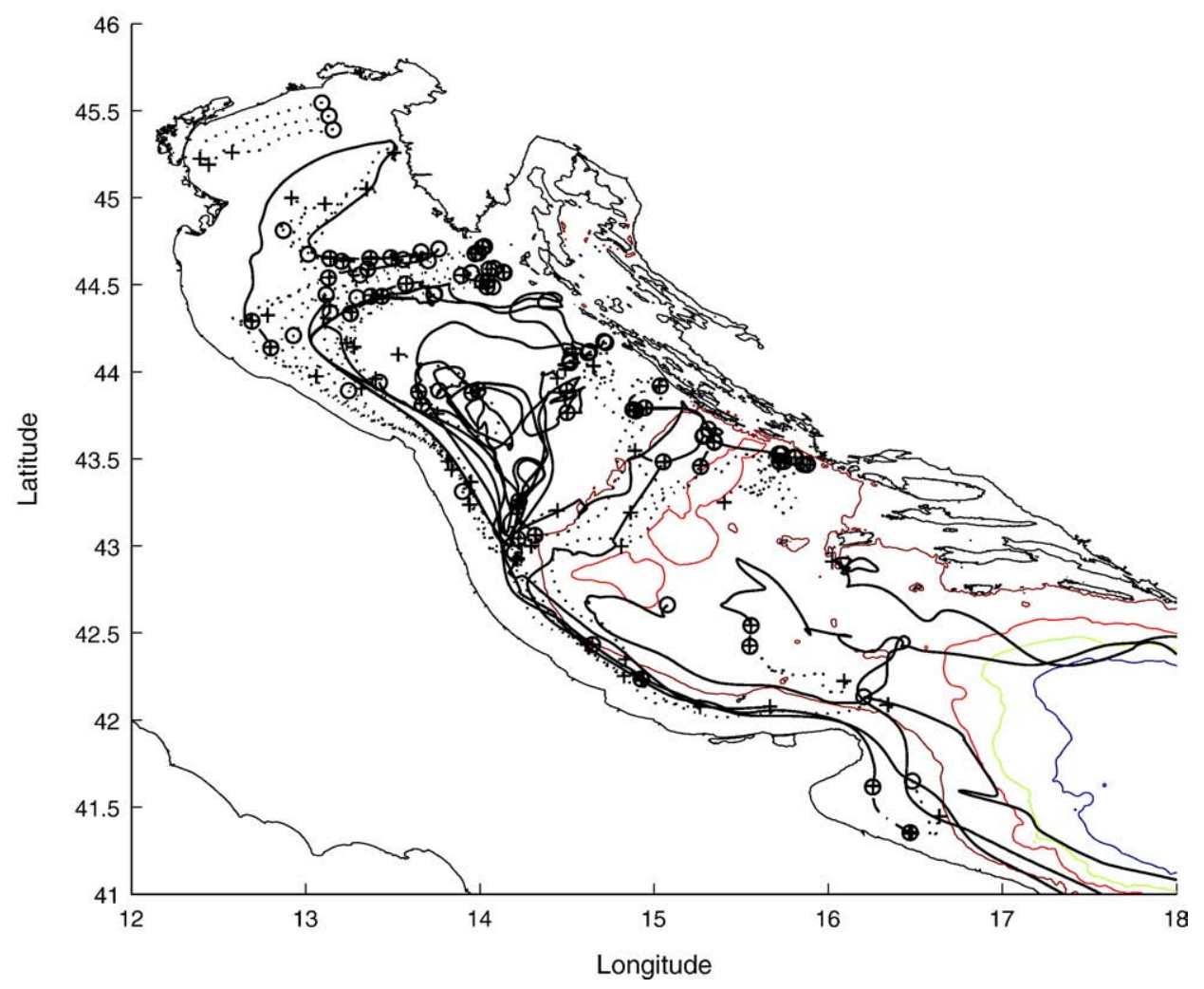

Fig. 1. Selected trajectories of drifters in the Adriatic Sea for the period January-February 2003. The dashed line shows the 48-hour track after 10Feb-2003. The dotted line shows the remaining track after that time. Circles and crosses indicate start and end position.

$00+09, \ldots 00+24)$. For further details, see Doms and Shattler (1999), Cacciamani et al. (2002) or the COSMO web site (http://www.cosmo-model.org).

\subsubsection{COAMPS model}

The Coupled Ocean/Atmosphere Mesoscale Prediction System (COAMPS) is a 3D finite difference, nonhydrostatic, sigma-coordinate model developed by the Naval Research Laboratory (Hodur, 1997). The version adopted was run in a re-analysis mode using three nested grids with the finest $4 \mathrm{~km}$ grid mesh centred over the Adriatic Sea. The two outer meshes are a $12 \mathrm{~km}$ grid covering the majority of the Mediterranean and a $36 \mathrm{~km}$ resolution European grid. The global NOGAPS model provides lateral boundary conditions for the $36 \mathrm{~km}$ grid at 6-hour intervals. In the reanalysis configuration, analyses are performed twice daily with forecasts for the following $15 \mathrm{~h}$. Forecast winds at 03, $06,09, \ldots 24 \mathrm{~h}(00+03,00+06,00+09,00+12,12+$ $03,12+06,12+09,12+12)$ were used. Details are documented in Hodur et al. (2001) for an evaluation of the COAMPS system and in Pullen et al. (2003) for the Adriatic re-analysis.

\subsubsection{SWAN model}

In order to simulate the wave characteristics, a thirdgeneration wave model, SWAN (Simulating WAves Nearshore), has been implemented for the Adriatic Sea with COAMPS wind forcing. The SWAN model was developed for shallow waters at Delft University Technology (TU Delft), with support from the Office of Naval Research (USA) and the Ministry of Transport, Public Works and Water Management (The Netherlands). The basic model used in this paper was SWAN version 4.41 .

Waves in SWAN are described with the two-dimensional wave action density spectrum. The equations take into account the local rate of change in time, the propagation in geographical space, the shifting of the relative frequency due to variations in depths and currents, and the depth-induced and current-induced refraction. The sink-source terms take into account the generation by wind, dissipation by white-capping, dissipation by depth-induced wave breaking, dissipation by bottom friction and redistribution of wave energy over the spectrum by non-linear wave-wave interactions. A full description of the SWAN model is given by 
Holthuijsen et al. (1989), Booij et al. (1999), Ris et al. (1999), and http://www.swan.ct.tudelft.nl.

Thirty-six uniformly distributed directions were used with 26 frequencies geometrically distributed: $f_{n+1}=$ $1.1 * f_{n}$, and $f_{1}=0.05 \mathrm{~Hz}$. The model time step was $10 \mathrm{~min}$ and the spatial grid had a uniform resolution of $2 \mathrm{~km}$ over the Adriatic. The bathymetry for the $2 \mathrm{~km}$ grid was interpolated from the finite element tidal model of Cushman-Roisin and Naimie (2002). The wind components from the wind models were linearly interpolated onto the $2 \mathrm{~km}$ wave model grid prior to running the simulations. Incoming waves at the open southeastern boundary of the Adriatic were assumed to be zero. The model was run in non-stationary mode with wave breaking enabled and Madsen bottom friction with default parameters.

Further details on these models and their respective implementations may be found in Signell et al. (2005).

Fig. 2 shows the model velocities before and during a BORA event for the ocean surface currents, Stokes drift from waves (e.g. Rixen et al., in press), wind velocities at $10 \mathrm{~m}$ and the drifter velocities optimally interpolated in space and time with $30 \mathrm{~km}$ spatial and 3 days temporal correlation lengths. LAMI shows weak winds on 8th February 2003 followed by strong BORA conditions on the 14th. ROMS surface currents and SWAN Stokes drift respond accordingly with strong NE-SW currents and Stokes drift components in the Northern Adriatic, and a strong signature of the Western Adriatic Current. These model outputs agree qualitatively with the surface drift as derived directly from the drifter data showing strong velocities in the Northern Adriatic and along the Western Adriatic coast from day 12 February onward. They have been interpolated on a common $5 \mathrm{~km}$ by $5 \mathrm{~km}$ grid.

Surface drift $48 \mathrm{~h}$ forecast errors from traditional approaches are presented in Fig. 3 for day 10 February (corresponding to day 12 February) and include simple ocean advection (top left), the rule of thumb imposing $3 \%$ of the wind magnitude rotated by $15^{\circ}$ to the right (top right), the combination of both (bottom left) and the subsequent addition of the Stokes drift (bottom right). ROMS ocean currents show larger discrepancies with drifter data along the Croatian coast and in the southern part of the basin probably due to a lack of drifter data used in the optimal interpolation. However, they seem to agree at least qualitatively with the gridded drifter data in the northern part along the Italian coast and during the strong Bora event in the northern Adriatic at day 14 February. This suggests that ROMS may be of some use to the hyper-ensemble, once corrected for its bias. The rule of thumb on the contrary shows large discrepancies along the Italian coast. The combination of the rule of thumb and ocean currents, and the subsequent addition of the Stokes drift contribution increase the errors further. Results for other days and lead times show qualitatively similar results.

\subsection{The hyper-ensemble}

Super-ensemble methods aim at combining models of the same kind (e.g. Krishnamurti et al., 2000a,b). This technique has been recently extended by Rixen and Ferreira-Coelho (in press) to the concept of hyperensemble, where models of different kinds are combined. Indeed, surface drift is a complex combination of a wide variety of processes.

Considering $\mathrm{N}$ models and $\mathrm{M}$ data, the simplest, linear combination of ocean and atmospheric models that matches the drifter tracks can be solved by minimizing Eq. (1) in a least-square sense if we allow for an independent term (otherwise, the last column and last weight are simply ignored).

$$
\left[\begin{array}{cccc}
v_{1,1} & . . & v_{1, N} & 1 \\
. . & . . & . . & . . \\
. . & . . & . . & . . \\
. . & . . & . . & . . \\
. . & . . & . . & . . \\
v_{M, 1} & . . & v_{M, N} & 1
\end{array}\right] *\left[\begin{array}{c}
w_{1} \\
. . \\
w_{N} \\
w_{N+1}
\end{array}\right]=\left[\begin{array}{c}
d_{1} \\
. \cdot \\
. \cdot \\
. . \\
. . \\
d_{M}
\end{array}\right]
$$

Here, $d$ represents the drifter velocities, $v$ represents the model velocities at the drifter data points and $w$ the weights to be optimized. The overall strategy is to find an optimal weighting of the ocean, atmospheric and wave models based on past or a priori information during a learning cycle at all grid points, and use them locally to compute new predictions in a forecast cycle, as a weighted combination of model forecasts for given lead times.

Negative regression weights $w$ can, and frequently do, occur in this type of formulation. This effect is typically caused by collinearities in the model forecasts. One may argue that the inclusion of a possible independent term/global bias in Eq. (1) does not have a physical meaning. However, we should bear in mind that the bias is a 'local property' of the hyper-ensemble. For specific purposes, the independent term is sometimes ignored to get a better understanding of the underlying physics.

Bearing in mind that the skills ('weights') are local properties of the super-ensemble, and considering that drifter data are Lagrangian and do not offer time series of velocity measurements at single points, one needs to 

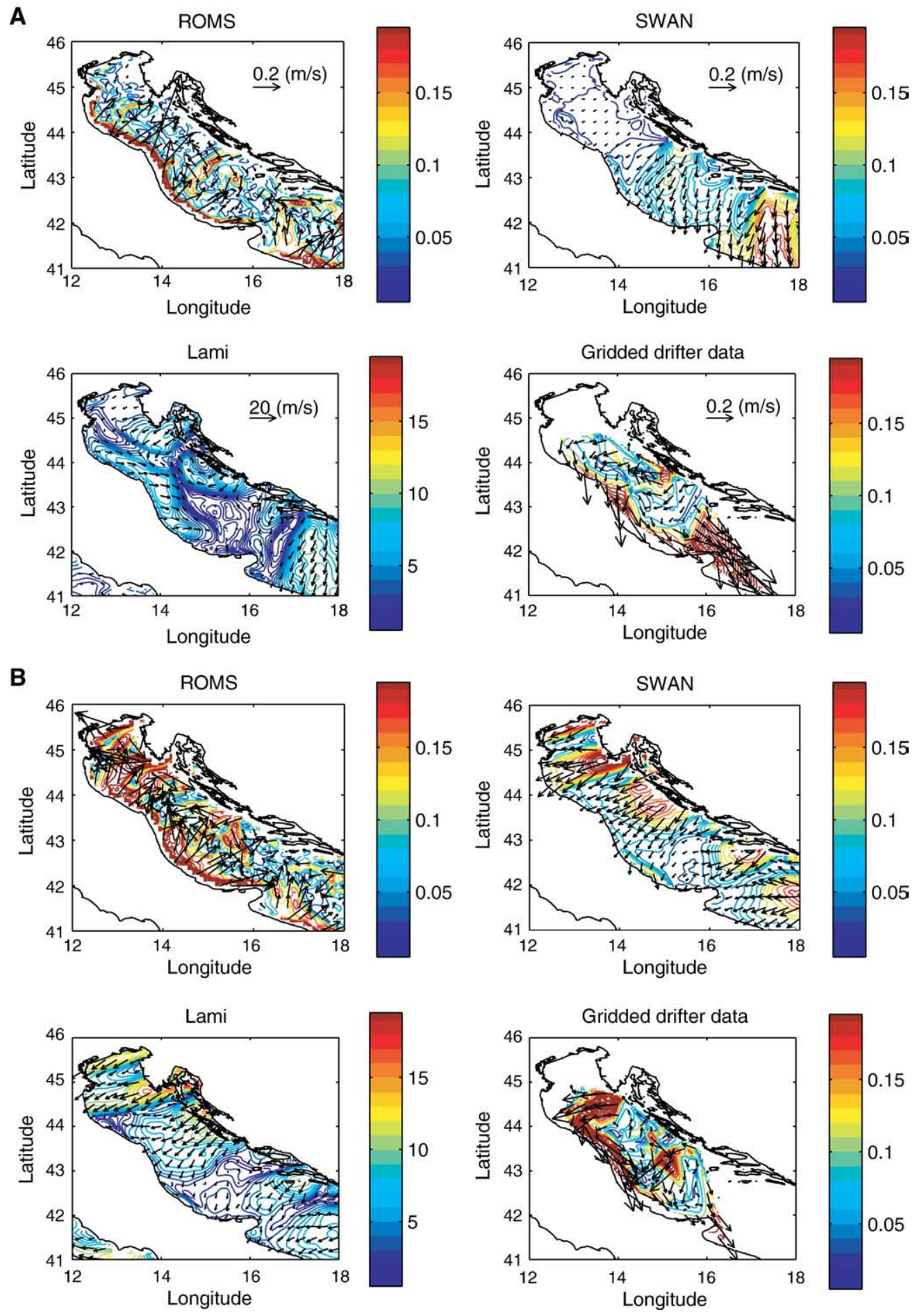

Fig. 2. ROMS ocean currents (top-left, surface), SWAN derived Stokes drift (top-right, surface), LAMI winds at 10 m (bottom-left) model nowcasts and gridded drifter velocities (bottom-right) (m/s) at days 8 (A) and 14 (B) February 2003, 00:00. The colorbar refers to the magnitude of the velocities contoured in the plots. 

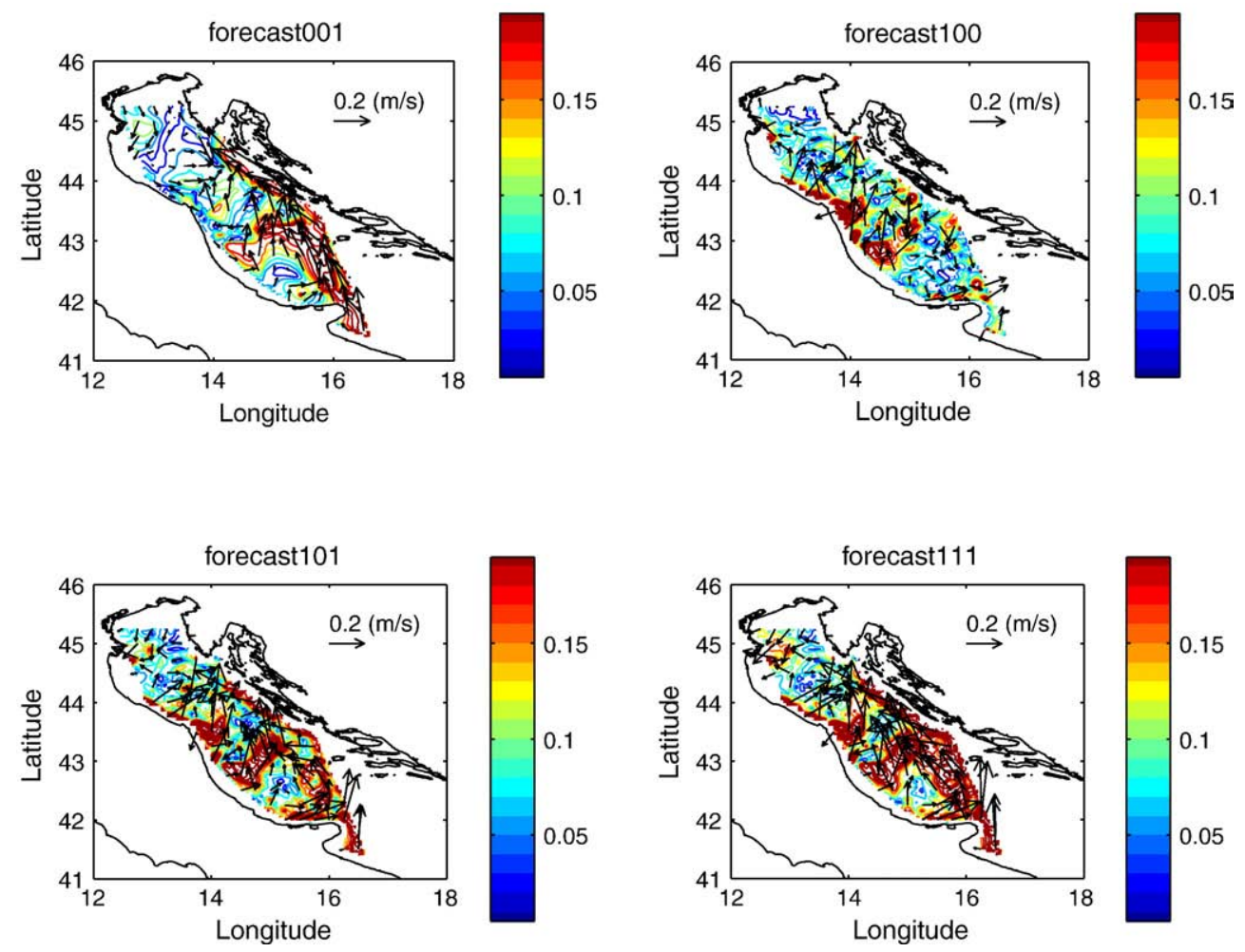

Fig. 3. Raw model surface drift $48 \mathrm{~h}$ forecast errors (m/s, vectors and magnitude in the color scale) at day 10 February 2003 corresponding to day 12 February 2003 for simple ocean advection (top left), the rule of thumb (top right), the combination of both (bottom left) and the subsequent addition of the Stokes drift (bottom right).

perform some kind of 4-D interpolation/objective analysis of the raw data, so that the linear regression described above can be performed at any single point on the common grid. On the contrary, in the field of meteorology, the linear regression would probably first be computed using the raw data time series at the meteorological stations to produce the local station forecast subsequently interpolated in space.

Of course, any number of available models might be considered in Eq. (1). When only one model is used (e.g. the ocean ROMS model only), the method reduces to a simple bias correction.

In the present study, this optimum is obtained using linear regression (with bias) in a least square sense with various learning periods, from 5 to 10, 25, 50 days. Here we assume local linear combinations of non-linear processes and neglect local non-linear interactions between wind, waves and currents, which would be beyond the scope of the present work and is a research topic on its own. For non-linear methods (neural networks, genetic algorithms, etc.) and further details on the hyper-ensemble strategy, we refer to Rixen and FerreiraCoelho (in press).

\section{Results}

Fig. 4 shows instantaneous $48 \mathrm{~h}$ forecast errors from day 10 February 2003, using unbiased ocean currents (top-left), unbiased rule of thumb (top-right), an unbiased combination of both (bottom-left), and the inclusion of the Stokes drift (bottom-right). In the case of the unbiased rule of thumb, the hyper-ensemble thus corrects for the angle and amplitude of the rule, besides the independent term. Errors remain similar among the different methods, larger along the coast, but generally speaking lower than the traditional forecast methods shown in Fig. 3. Results for other days, lead times and combinations of models show qualitatively similar results.

Local RMS forecast errors provide a simple measure of the uncertainty associated with the traditional methods (Fig. 5) and hyper-ensemble methods (Fig. 6) corresponding to Figs. 3 and 4 respectively. These errors are computed over a $48 \mathrm{~h}$ period around day 12 February 2003. Although the ocean currents had lower instantaneous errors (Fig. 3, top-right), the uncertainty of the rule of thumb is lower than any other standard method. 

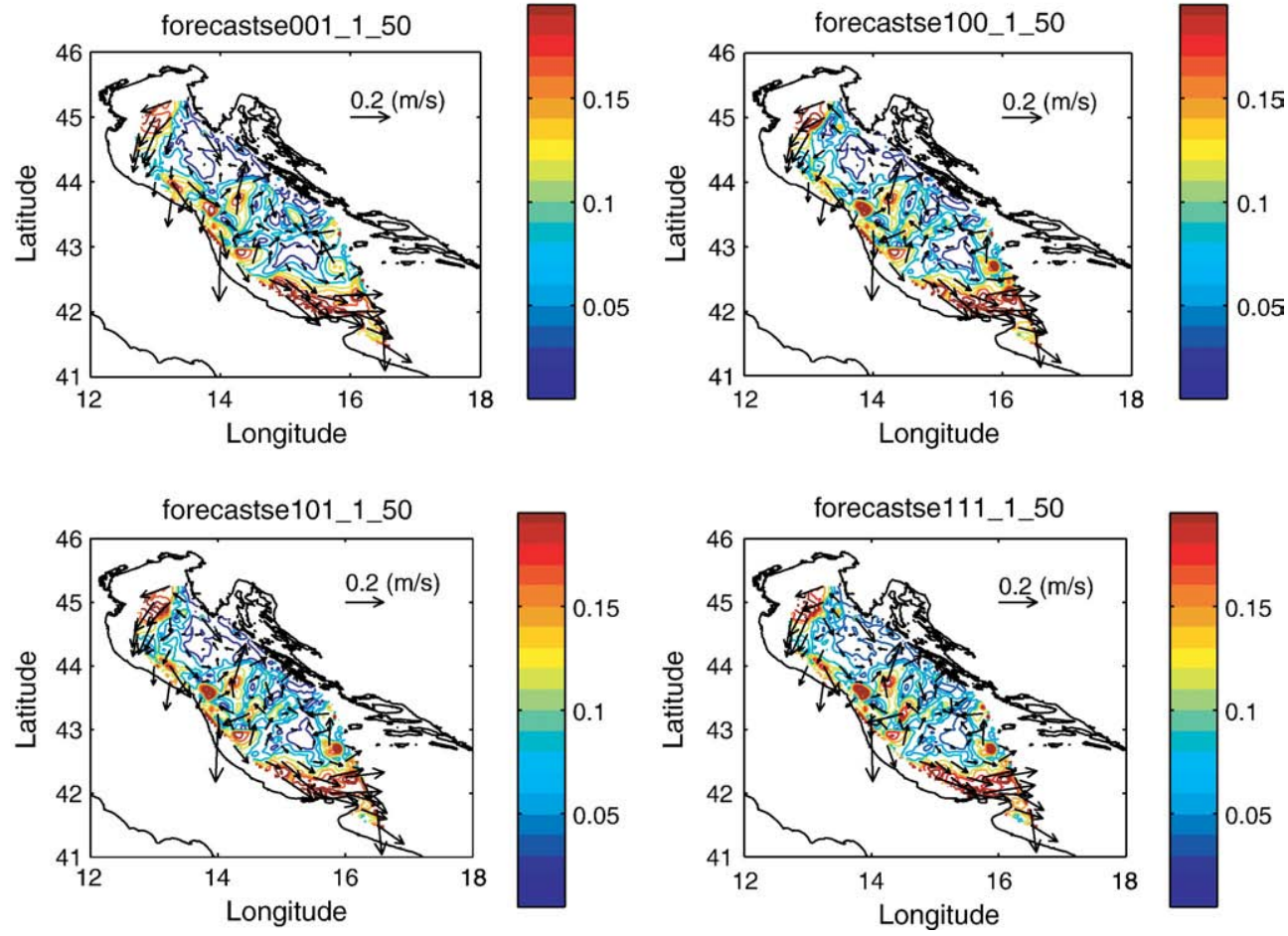

Fig. 4. Hyper-ensemble surface drift $48 \mathrm{~h}$ forecast error (m/s, vectors and magnitude in the color scale) at day 10 February 2003 corresponding to day 12 February 2003 with a 50 days learning period, using locally unbiased ocean advection (top left), bias corrected rule of thumb (top-right), the unbiased combination of both ocean and atmospheric models (bottom left) and the subsequent addition of the Stokes drift in the hyper-ensemble (bottom right).
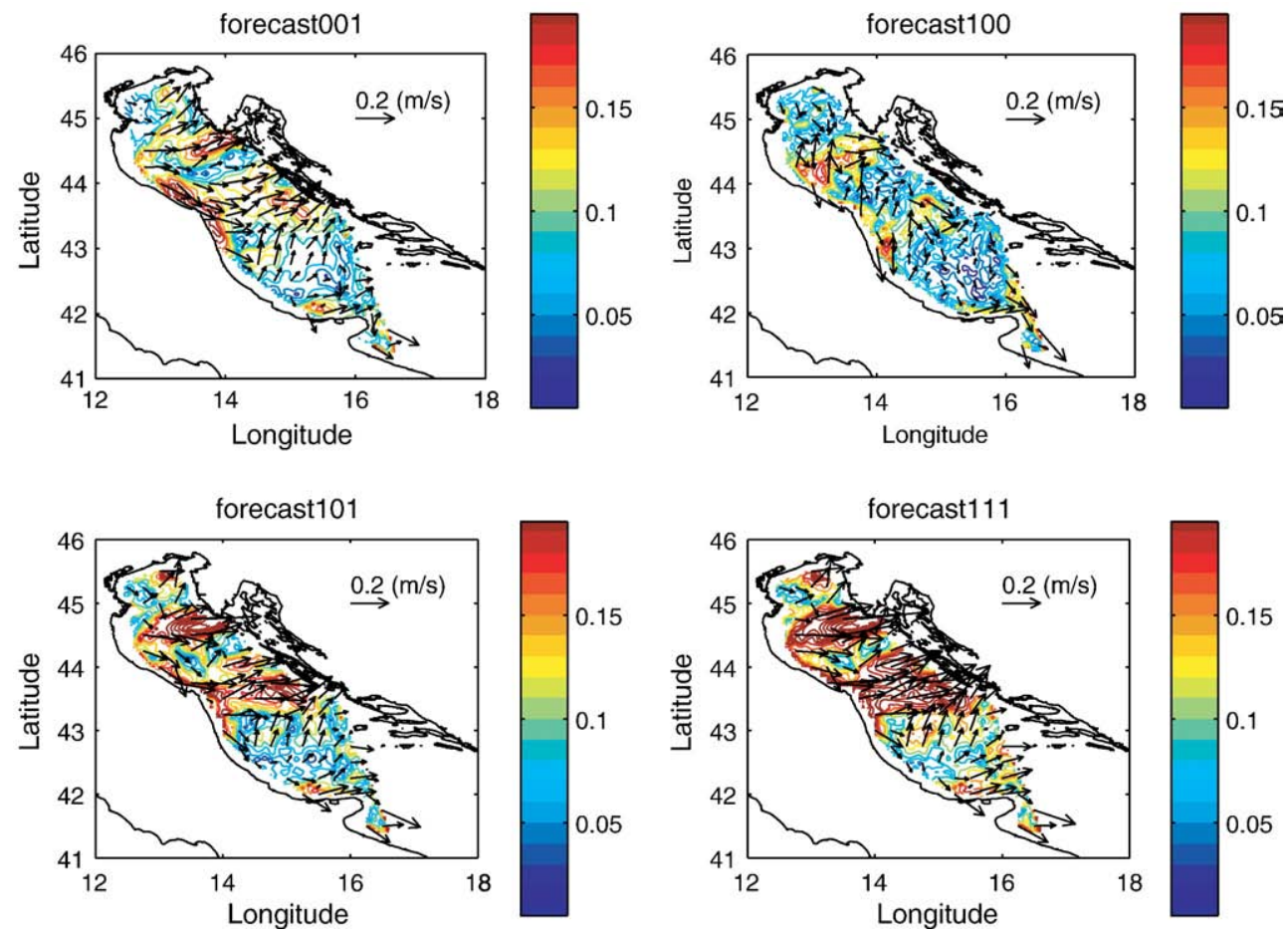

Fig. 5. Local RMS forecast error (m/s, vectors and magnitude in the color scale) corresponding to traditional methods depicted in Fig. 3. 

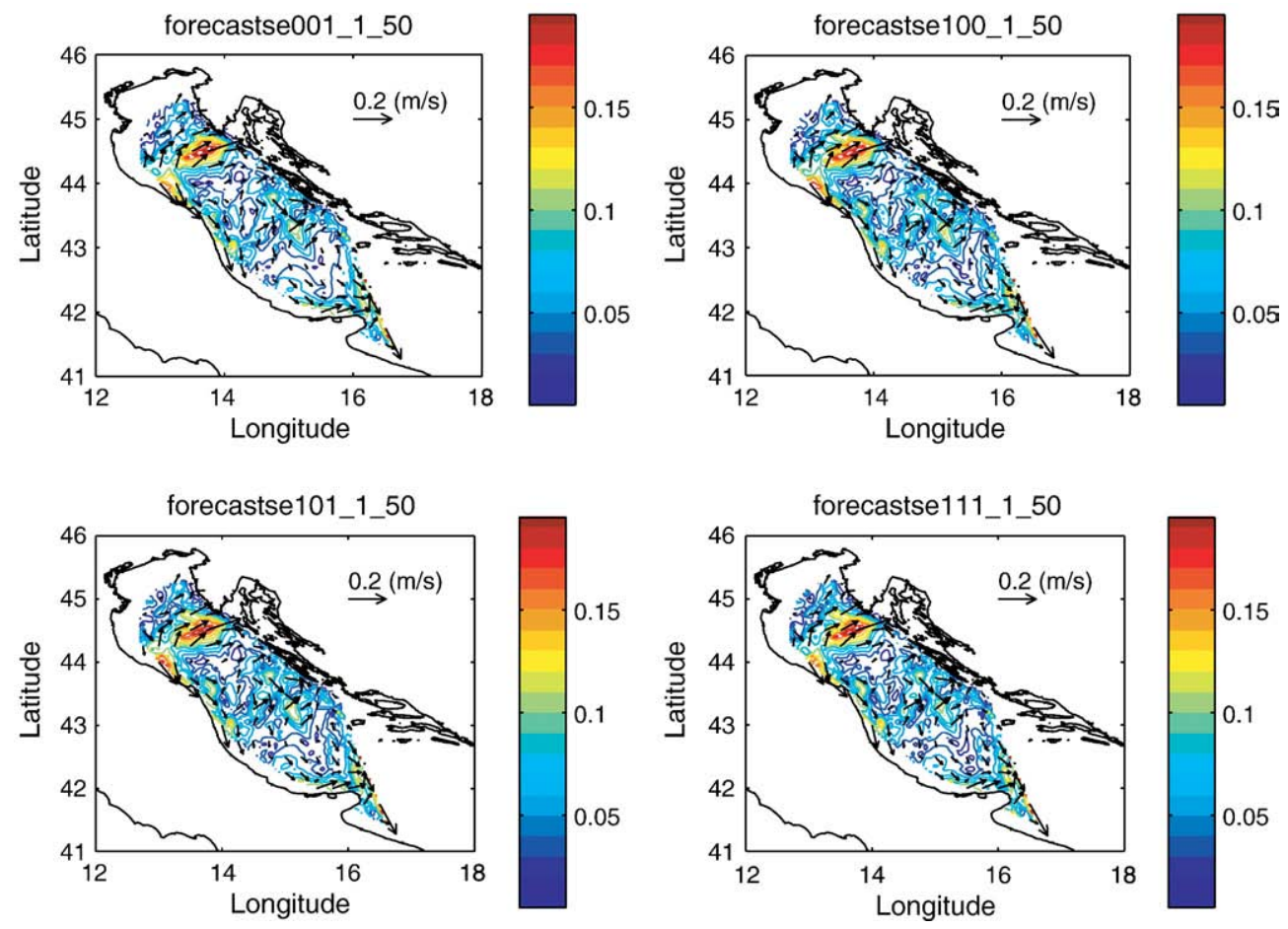

Fig. 6. Local RMS forecast error (m/s, vectors and magnitude in the color scale) corresponding to hyper-ensemble methods depicted in Fig. 4.

The corresponding RMS forecast errors for the hyperensemble methods (Fig. 6) show an overall reduction of the uncertainty. The consistency throughout the different methods suggests that the correction is essentially a bias correction. The combination of different models has a minor impact on the hyper-ensemble skills. Again, results for other days, lead times and combinations of models show qualitatively similar results.

At this stage, some wider statistical analyses are needed to compare quantitatively the skills of the different surface drift forecasts. Fig. 7 shows some standard statistics for the different methods on the two components of velocity. In hindcast mode, the bias should ideally vanish: statistics are compared here to the true drifter values instead of the interpolated values. Hence some minor bias remain. Statistics are consistent between the hindcast and the forecast, implying that the weights remain roughly valid and useful in predicting surface drift. The rule of thumb (the second bar) remains usually the more robust of the traditional methods but is outperformed by the hyper-ensemble methods, especially for long training periods (50 days) in the hyper-ensemble using all models (last bar). Again, results for other days, lead times and hyperensemble combinations of models show qualitatively similar results.
Uncertainty maps as derived previously from the recent $48 \mathrm{~h}$ forecast may be used to associate twodimensional probability distribution areas with single drifter tracks instances. Ensembles of 100 members were generated by a random walk procedure, adding Gaussian velocities with standard deviation equal to the uncertainty velocities derived as above, from which encompassing convex hulls of $100,75,50$ and $25 \%$ of the end position are identified with a shrinking of the convex hull shape down to the mean position of the ensemble. Several examples are illustrated in Fig. 8. The persistence might provide useful and quite robust information if the drifter is not undergoing a radical direction change just afterwards. Ocean currents are overestimated and potentially in a wrong direction. The rule of thumb method in these examples is not very robust either. Individual models are hindered by local biases and limited process representation. Ocean models, for example, do usually not take the Stokes drift into account. Only the hyper-ensemble solutions have tracks similar to the observed drifters. Their probability distribution areas sometimes capture the true drifter end position, which is not the case for the standard methods.

The assumption of normality of the error probability distribution function may be disputed. Results show that 

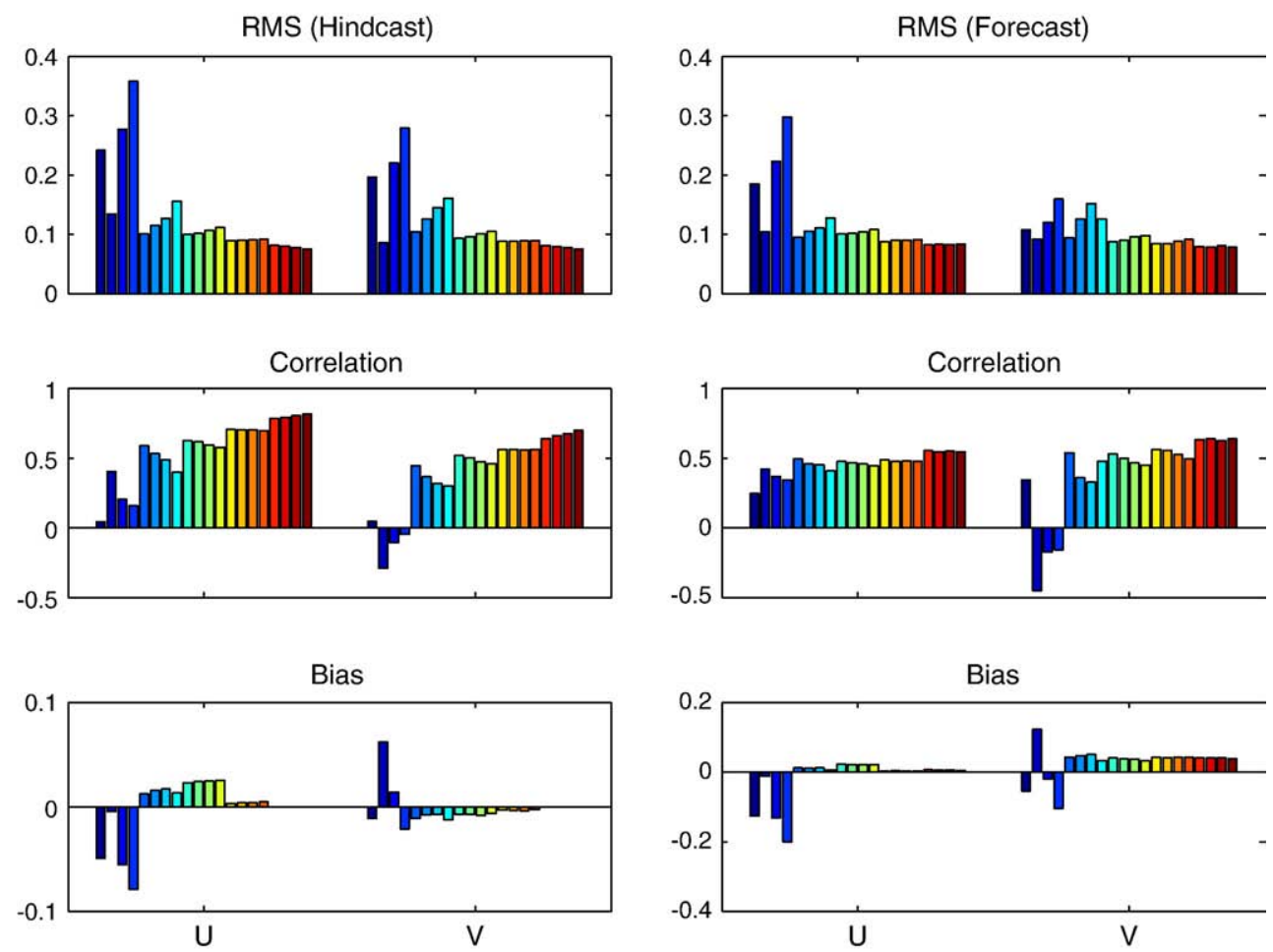

Fig. 7. Hindcast and $48 \mathrm{~h}$ forecast RMS errors $(\mathrm{m} / \mathrm{s})$, correlation and bias $(\mathrm{m} / \mathrm{s})$ on U and $\mathrm{V}$ components of velocity for the different methods for day 10 February 2003 corresponding to 12 February 2003. Bars from left to right represent respectively: (1) ocean currents, (2) wind rule of thumb, (3) the addition of (1) and (2), (4) the addition of the Stokes drift, and the corresponding hyper-ensemble combinations for a 5 (bars 5-8), 10 (bars $9-12$ ), 25 (bars 13-16) and 50 (bars 17-20) days learning period.

the single realization and the ensemble 'centroid' track are in relative good agreement in many cases. The limitation of this hypothesis however becomes obvious in cases where the single realization (not part of the ensemble) end position is not within the ensemble $25 \%$ or $50 \%$ probability polygon area.

Fig. 9 summarizes the reliability of the estimation of the probability distribution areas for the different methods. The traditional methods (first 4 bars) provide unreliable end positions, far from the true position and almost a null probability of capturing the true position in the ensemble convex hull. On the contrary, the hyperensemble end positions, ensemble histogram maximum (in $5 \mathrm{~km} * 5 \mathrm{~km}$ bins) and gravity center are much closer to the true drifter end position. The results are slightly better for shorter learning period, which contradicts somewhat the optimal 50 days found previously. The probability of capturing the true end position in the ensemble convex hull is usually 0 for the traditional methods and ranges from $20 \%$ to $35 \%$ for the hyperensemble solutions.

\section{Discussion and conclusions}

The hyper-ensemble approach is a very generic tool for geophysical applications. This statistical approach makes the best use of all available data, with a marginal effort, provided that underlying models and data are available.

The application of the hyper-ensemble technique in the challenging area of the Adriatic Sea during a Bora event has shown significant improvements in surface drift forecast, both on field estimates, integrated drifter tracks and probability distribution area estimation. However, results also suggest that this technique is still very far from a very reliable surface drift forecasting system that may be used in search- and-rescue cases or dramatic oil spill incidents.

Two major limitations of the existing surface drift approaches can be identified which deserve attention in future developments.

On the one hand, comprehensive observational networks are needed to cover the spatio-temporal and spectral range of processes found in a specific area. 


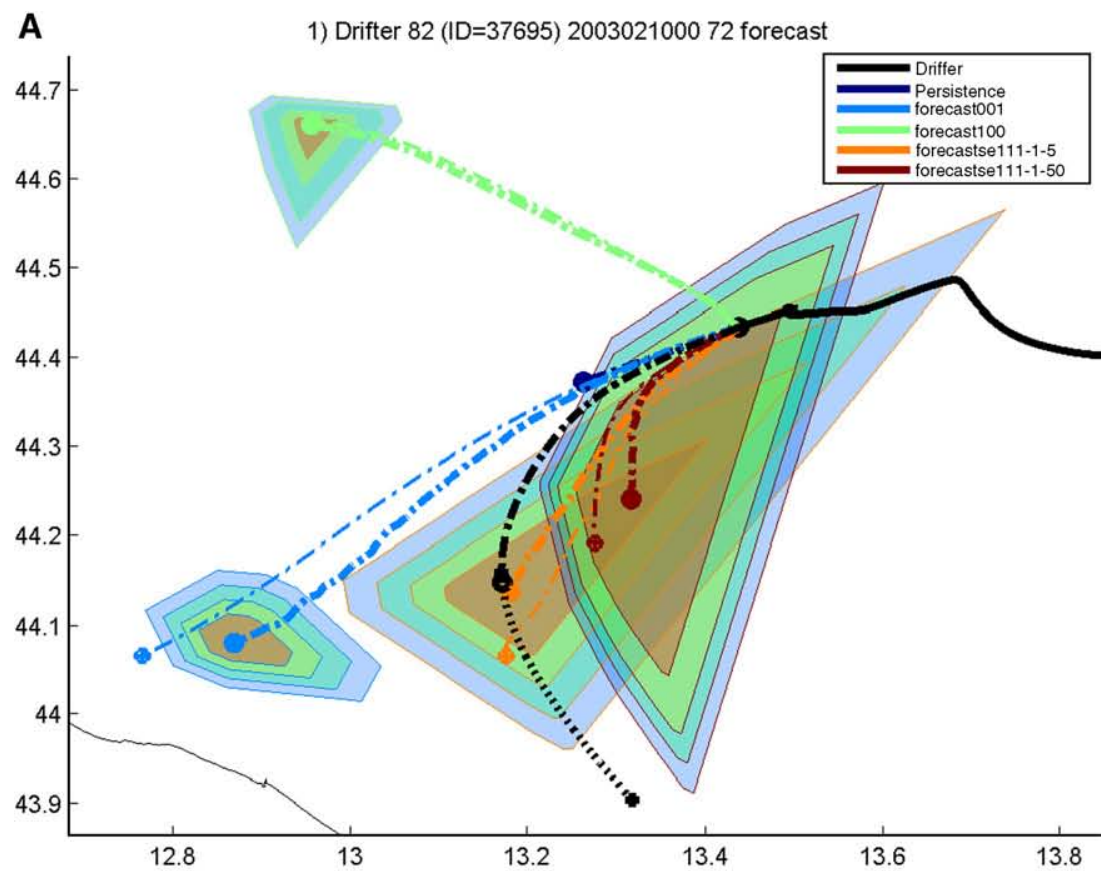

\section{B}

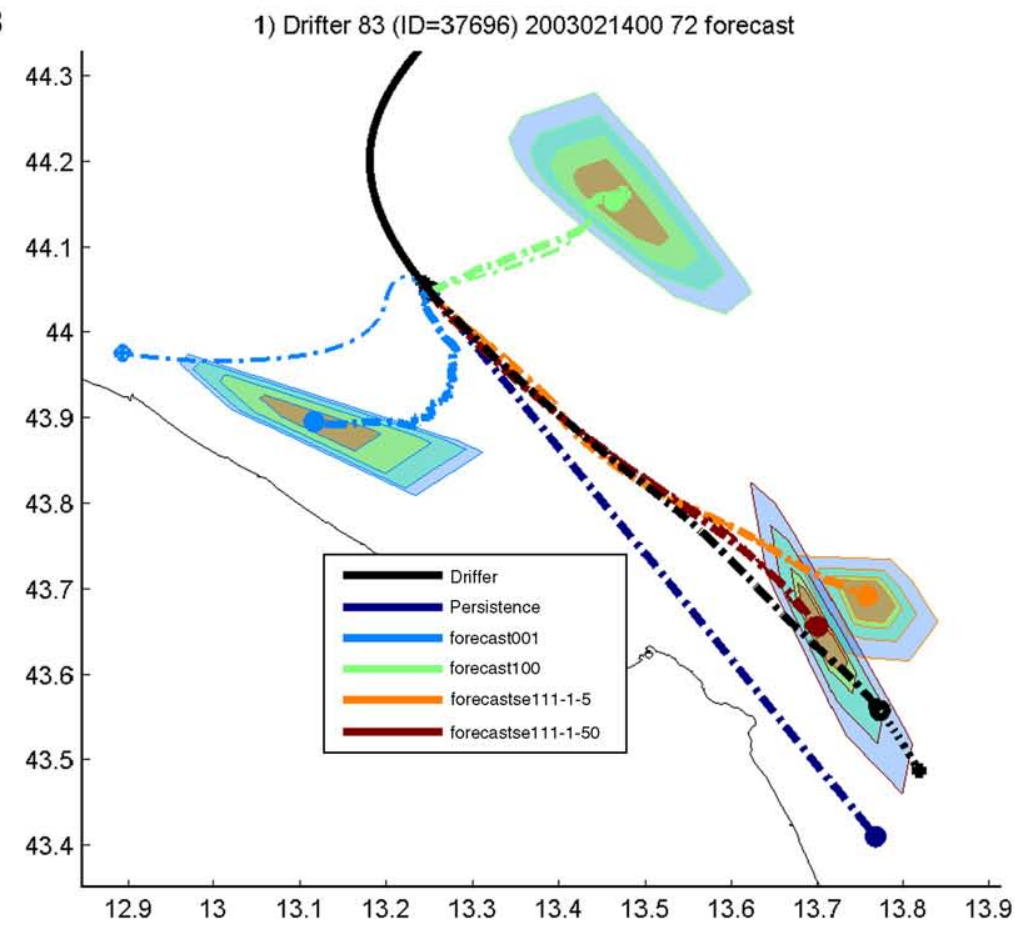

Fig. 8. Some examples of drifter tracks (past - continuous line, $72 \mathrm{~h}$ forecast - dashed line), single realization (thin line), ensemble track (thick line) and associated probability distribution areas for different methods: true drifter track (black), persistence (dark blue), ocean current (blue), rule of thumb (green), hyper-ensemble with all models using 5 days (orange) and 50 days (brown) learning period. Convex hulls in decreasing order represent the estimation of the $100,75,50$ and $25 \%$ probability distribution areas corresponding to the different methods. 


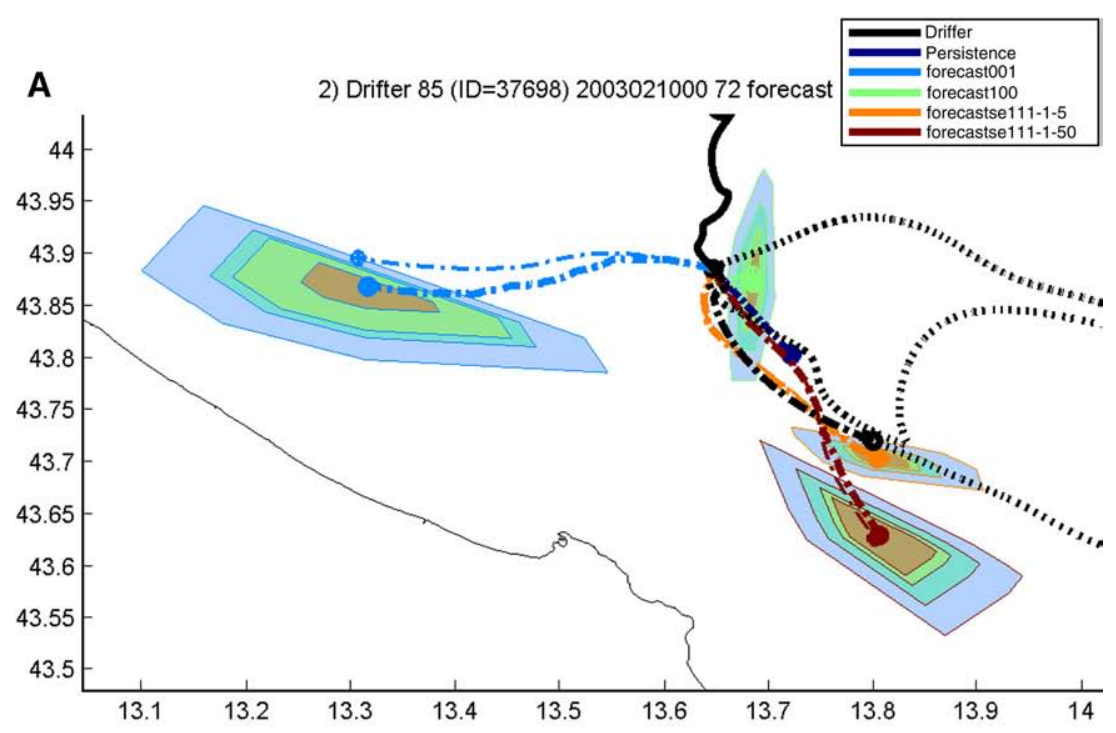

B

1) Drifter 93 (ID=37708) 200302060072 forecast

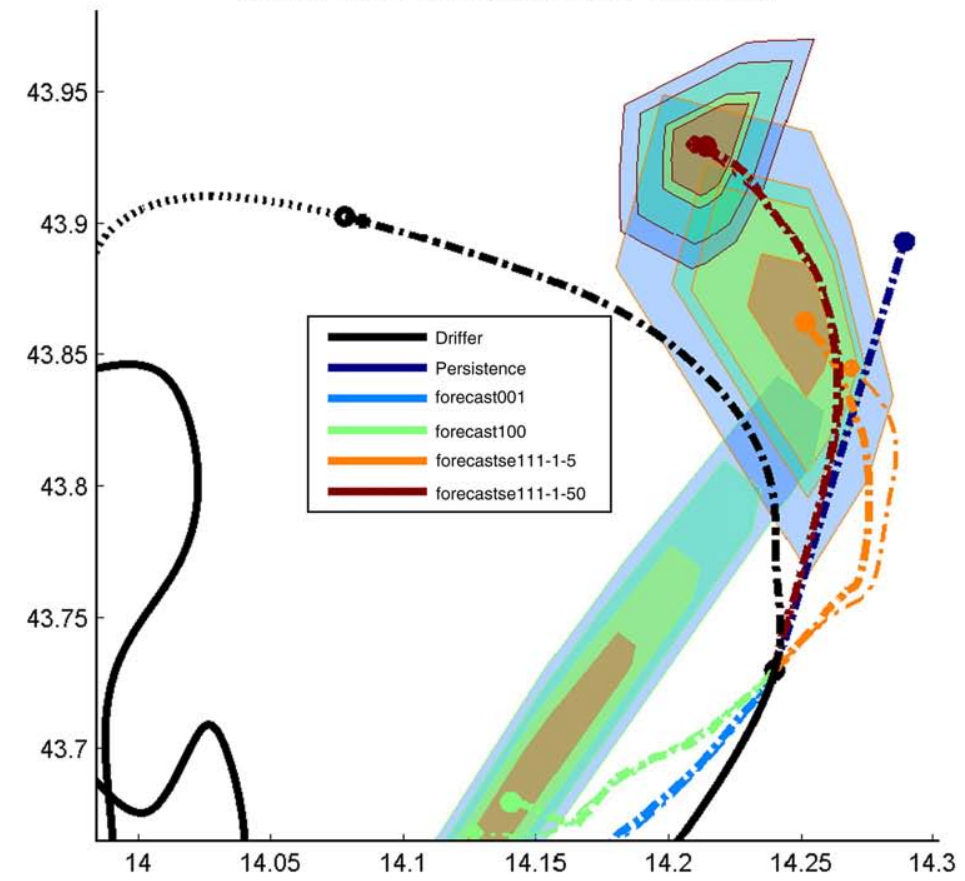

Fig. 8 (continued).

Super- or hyper-ensembles provide a direct feedback to modelers on local performance of their respective models by identifying local weaknesses or strengths of models and areas where local data assimilation might have a significant benefit on the individual and ensemble models. The amount and nature (i.e. Lagrangian vs. Eulerian) of data available will determine the super-ensemble approach to be used. Lagrangian data imply a prior objective analysis of available data or the assumption of some local homogeneous area. Eulerian times series of data on the contrary offer the advantage of an easier implementation of the hyper-ensemble method, but require usually expensive data collection at sea with dedicated instrumentation.

On the other hand, individual models also require further improvement in their respective forecast skills. 
200302140048
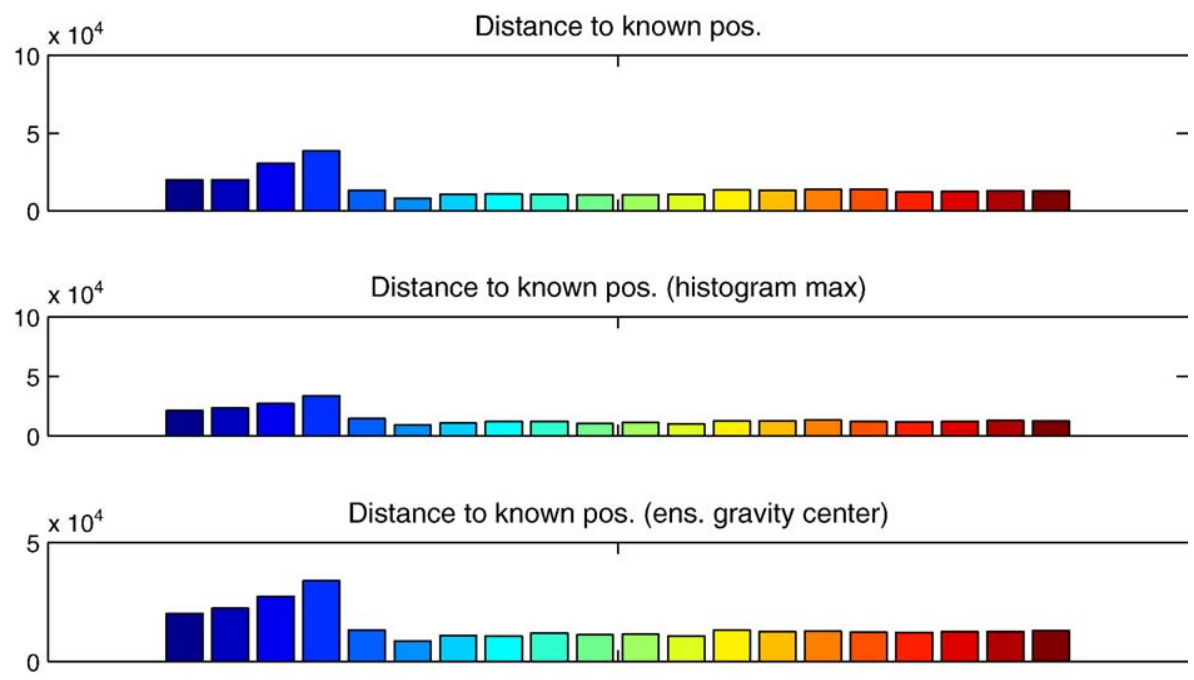

Prob. of success (true pos. in ens. conv. hull)

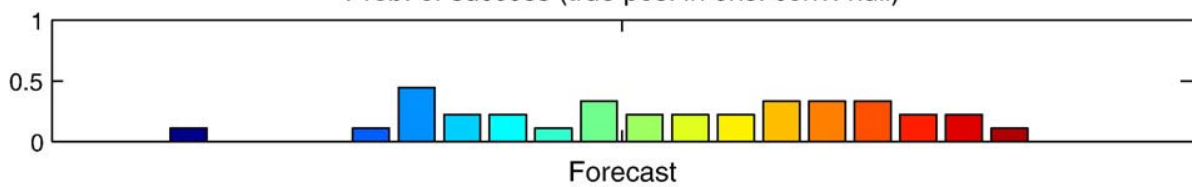

Fig. 9. Summary of reliability of the estimation of probability distribution areas for the different methods (as in Fig. 7 ) for $48 \mathrm{~h}$ forecast on day 14 February: (top) mean distance of single instance end position to the true end positions; (top-middle) mean distance of ensemble end position histogram maximum to the true end positions; (bottom-middle) mean distance of ensemble gravity center of end positions to the true positions; (bottom) probability that the convex hull captures the true end position.

We have seen that individual models still have great difficulties producing accurate surface drift velocities.

Only a joint observational and modelling effort may improve directly the hyper-ensemble approaches which require both components. The consistency between the different hyper-ensemble combinations has shown that the major correction arises from the local bias removal. Only a marginal improvement can be expected from the inclusion of two or more processes in the hyperensemble. Indeed, all ocean, wave and atmospheric models are usually strongly correlated (eventually disregarding the rotation), as a result of the atmospheric forcing on the other two components, with the exception of the western Adriatic coast, where the coastal jet is, defacto, probably less sensitive to wind forcing because of the strong advection.

\section{Acknowledgments}

This work was performed at NURC and funded by the North Atlantic Treaty Organization. All people who contributed to the observational and modelling effort during the ADRIA02 and ADRIA03 field experiments are deeply acknowledged. We are grateful to Pierre-Marie Poulain for processing and providing the drifter data.

\section{References}

Booij, N., Ris, R.C., Holthuijsen, L.H., 1999. A third-generation wave model for coastal regions. Part I-Model description and validation. Journal of Geophysical Research 104 (C4), 7649-7666.

Cacciamani, C., Emiliani, P., Ferri, M., Minguzzi, E., 2002. High resolution verification of hydrostatic and non-hydrostatic LAM precipitation forecasts in Italy. In: Doms, G., Shatter, U. (Eds.), COSMO Newsletter, vol. 176, $\mathrm{n}^{\circ} 2$. Deutscher WetterDienst (DWD), Offenbach, Germany, p. 186. February 2002.

Carniel, S., Umgiesser, G., Sclavo, M., Kantha, L., Monti, S., 2002. Tracking the drift of a human body in the coastal ocean using numerical prediction models of the oceanic, atmospheric and wave conditions. Science \& Justice 42 (3), 143-151.

Cushman-Roisin, B., Naimie, C.E., 2002. A finite element model of the Adriatic tides. Journal of Marine Systems 37 (4), 279-297.

Doms, G., Shattler, U., 1999. The Nonhydrostatic Limited-Area Model LM (Lokal-Modell) of DWD. Part I: Scientific Documentation. Deutscher WetterDienst (DWD), Offenbach, Germany. 
Gill, A.E., 1982. Atmosphere-Ocean Dynamics. Academic Press. 662 $\mathrm{pp}$.

Hodur, R.M., 1997. The Naval Research Laboratory Coupled Ocean/ Atmosphere Mesoscale Prediction System (COAMPS). Monthly Weather Review 125 (7), 1414-1430. doi:10.1175/1520-0493.

Hodur, R.M., Pullen, J., Cummings, J., Hong, X., Doyle, J.D., Martin, P., Rennick, M.A., 2001. The Coupled Ocean/Atmosphere Mesoscale Prediction System (COAMPS). Oceanography 15, 88-98.

Holthuijsen, L.H., Booij, N., Herbers, T.H.C., 1989. A prediction model for stationary, short-crested waves in shallow water with ambient currents. Coastal Engineering 13, 23-54.

Kovacevic, V., Gacic, M., Poulain, P.-M., 1999. Eulerian current measurements in the Strait of Otranto and in the Southern Adriatic. Journal of Marine Systems 20, 255-278.

Krishnamurti, T., Kishtawal, C., Zhang, Z., Larow, T., Bachiochi, D., Williford, E., 2000a. Multimodel ensemble forecasts for weather and seasonal climate. Journal of Climate 13, 4196-4216.

Krishnamurti, T., Kishtawal, C., Shin, D., Williford, E., 2000 b. Improving tropical precipitation forecasts from a multianalysis superensemble. Journal of Climate 13, 4217-4227.

Kumar, T.V., Krishnamurti, T., Fiorino, M., Nagata, M., 2003. Multimodel superensemble forecasting of tropical cyclones in the Pacific. Monthly Weather Review 131, 574-583.

Majewsky, D., 1998. The new global icosahedral-hexagonal grid point model GME of the Deutscher Wetterdienst. Recent Developments in Numerical Methods for Atmospheric Modelling, ECMWF Seminar Proceedings, pp. 173-201.

Majewsky, D., Liermann, D., Prohl, P., Ritter, B., Buchhold, M., Hanisch, T., Paul, G., Wergen, W., Baumgardner, J., 2002. The operational global icosahedral-hexagonal gridpoint model GME: description and high resolution tests. Monthly Weather Review 130, 319-338.

Poulain, P.-M., Mauri, E., Zanasca, P., 2003. Upper thermal structure and intermediate currents in the Adriatic and Northern Ionian as measured by a Lagrangian profiler in 1995 and 1996. Bollettino di Geofisica Teorica ed Applicata 44 (2), 139-154.

Pullen, J., Doyle, J., Hodur, R., Ogston, A., Book, J., Perkins, H., Signell, R.P., 2003. Coupled ocean-atmosphere nested modeling of the Adriatic Sea during winter and spring 2001. Journal of Geophysical Research 108 (C10), 3320. doi:10.1029/2003JC001780.

Ris, R.C., Booij, N., Holthuijsen, L.H., 1999. A third-generation wave model for coastal regions. Part II - verification. Journal of Geophysical Research 104 (C4), 7667-7681.

Rixen, M., Ferreira-Coelho, E., 2005. Operational prediction of acoustic properties in the ocean using multi-model statistics. Ocean Modelling 11 (3-4), 428-440. doi:10.1016/j.ocemod.2005.02.002.

Rixen, M., Ferreira-Coelho, E., in press. Operational surface drift prediction using linear and non-linear hyper-ensemble statistics on atmospheric and ocean models. Journal of Marine Systems. doi:10.1016/j.jmarsys.2004.12.005.

Shin, D., Krishnamurti, T., 2003a. Short- to medium-range superensemble precipitation forecasts using satellite products: 1 . Deterministic forecasting. Journal of Geophysical Research 108 (8), 8383. doi:10.1029/2001JD001510.

Shin, D., Krishnamurti, T., 2003b. Short- to medium-range superensemble precipitation forecasts using satellite products: 2 . Probabilistic forecasting. Journal of Geophysical Research 108 (8), 8384. doi:10.1029/2001JD001511.

Signell, R.P., Carniel, S., Cavaleri, L., Chiggiato, J., Doyle, J.D., Pullen, J., Sclavo, M., 2005. Assessment of wind quality for oceanographic modelling in semi-enclosed basins. Journal of Marine Systems 53 (1-4), 217-233.

Umlauf, L., Burchard, H., 2003. A generic length-scale equation for geophysical turbulence model. Journal of Marine Research 61, $235-265$. 